\title{
Correlation Analysis of Car Exterior and Interior Noise Tests under Different Yaw Angles with Beamforming
}

\author{
Wenyu Jia ${ }^{1,2, a}$, Tao Zhang ${ }^{1,2, b}$, Guoxu Dong ${ }^{1,2, c}$, \\ Bin Wang ${ }^{3, \mathrm{~d}}$ and Yinzhi $\mathrm{He}^{3, \mathrm{e}}$ \\ ${ }^{1}$ Chongqing Changan Automobile Co. Ltd., Qongqing, 404100, China \\ ${ }^{2}$ State Key laboratory of Vehicle NVH and Safety Technology, Qongqing, 404100, China \\ ${ }^{3}$ Shanghai Automotive Wind Tunnel Center, Tongji University, Shanghai, 201804, China \\ ajiawy@changan.com.cn, bzhangtaoathuda2010@126.com, cdongguoxu@126.com, \\ 'wbin@tongji.edu.cn, 'eyinzhi.he@sawtc.com
}

Keywords: Beamforming; Correlation analysis; Yaw angle; Wind tunnel.

Abstract. To identify vehicle wind noise sources in aeroacoustic wind tunnel, Beamforming technique is widely used today. In this study, a production car Roewe 350 was set on the turntable at 1:1 aeroacoustic wind tunnel of Shanghai Automotive Wind Tunnel Center (SAWTC), a planar spiral microphone array with 120 channels $\left(1.8 \mathrm{~m}^{*} 1.8 \mathrm{~m}\right)$ was set out-of-flow to test its exterior wind noise sources. In interior of the vehicle near the left side view mirror triangle and driver's ear position, 2 reference microphones were set to record potential sound source signal. The results demonstrate that Beamforming technique is a practical method for sound source identification. Furthermore, correlation analysis makes it clear, that side view mirror contributes most to vehicle interior noise. Under different yaw angles, exterior noise sources distribution is different, so is the correlation result.

\section{Introduction}

Noise produced from motor vehicles basically comprises drivetrain noise, tire-road noise and wind noise. For a sedan, as driving speed increases above $100 \mathrm{~km} / \mathrm{h}$, wind noise can play a dominant role, which becomes an important fatigue factor for drivers and passengers on a long trip [1]. Therefore, it is important to study vehicle exterior noise sources under wind excitation and their transmission path to vehicle interior, where noise level is to be optimized as target.

With the development of Beamforming technique in last decades, Microphone array becomes a powerful tool for sound source localization in vehicle development [2]. In this article, with a microphone array set out- of -flow and combined with two reference microphones inside the vehicle, correlation function between array signals and each reference microphone can be calculated, so that the contribution of exterior noise source to interior can be found out. Under different Yaw angles, vehicle exterior noise sources distribution varies and correlation function changes accordingly.

\section{Beamforming Theory}

In this study, Beamforming theory is mainly used for time domain sound source identification and correlation analysis, including:

Time Domain Beamforming and HDR. Time domain Beamforming is based on the theory of delay and sum and can be written as follows [3]:

$\hat{f}\left(x_{i} t\right)=\frac{1}{M} \sum_{i=1}^{M} w_{i} f_{i}\left(x_{i}\left(t-\Delta_{i}\right)\right)$

$f(x, t)$ : Calculated time function on location $x$ of the map;

$M:$ Number of microphones; 
$w_{i}:$ Weighting factor for microphone signal i;

$\Delta_{i}$ : Time delay for microphone signal i to location $x$ on the Beamforming map.

HDR (Hign dynamic Range) is an advanced beamforming algorithm based on acoustic eraser. At the beginning, the mainlobes are successively extracted from the signal and the sidelobes are suppressed, at last all the extracted sources are to be combined in an acoustic map, in which way the available dynamic range of the array can be significantly enlarged.

Beamforming Using a Reference Signal. The purpose of this study is to investigate the locations of exterior noise sources and their contribution to vehicle interior under different conditions. Therefore, cross correlation functions are to be applied. In the time domain, according to eq.2, cross correlation function $C(\tau)$ between reference signal $\mathrm{F}_{\mathrm{R}}(t)$ and the series of microphone signals Fi $(t)$ of array can be calculated [4]:

$$
C(\tau)=\frac{\int_{-\infty}^{\infty} F_{R}(t) F_{i}(t+\tau) d t}{\sqrt{\left\|F_{R}(t)\right\| \cdot\left\|F_{i}(t)\right\|}}
$$

With $C(\tau)$ the contribution of exterior source to vehicle interior noise can be detected.

\section{Test Conditions and Measurement Setup}

For the test conditions, all measurements were performed on a production sedan Roewe350, which was set at the full scale aeroacoustic wind tunnel (AAWT) of SAWTC at Tongji university. This wind tunnel is Goettingen type equipped with a $3 / 4$ open-jet, closed test section with nozzle area $27 \mathrm{~m}^{2}$. Background noise measured out-of-flow is less than $61 \mathrm{dBA}$ at the wind speed $160 \mathrm{~km} / \mathrm{h}$ [5].

All tests were carried out with the incoming flow at a speed of $120 \mathrm{~km} / \mathrm{h}$. With the help of turntable, the vehicle was tested under different yaw angles of $0^{\circ},+20^{\circ}$ and $-20^{\circ}$ to investigate the influence of crosswind during driving on the road. The definition of yaw angle at AAWT of SAWTC is as follows: yaw angle $+20^{\circ}$ means that test vehicle rotates clockwise on the balance turntable, makes driver position windward, and the front passenger position on the leeside. Inversely, yaw angle $-20^{0}$ means that test vehicle rotates anticlockwise, makes driver position on the lee side, and the front passenger position windward.

All tests of the vehicle were conducted under the standard baseline condition as originally supplied. During the test procedure HVAC system of vehicle was closed and put on the recirculation mode, all HVAC outlets were closed as well, in order that no wind could blow into the vehicle interior directly. Meanwhile the boundary layer suction system and the 5-running belts system were not in operation. Additionally, the test temperature was about $22-24^{\circ} \mathrm{C}$, relative humidity $36-40 \%$ and ambient air pressure 103.2-103.5kPa.

For the acoustic measurements, basically the "acoustic camera" system made from Gfaitech GmbH was used. During the test, a 120-channel planar spiral array with dimension of $1.8 \mathrm{~m} * 1.8 \mathrm{~m}$ was set out-of-flow (Fig.1), and the distance between this array and the front left side glass of the car is $3.85 \mathrm{~m}$. At the same time the camera in the middle of the array was directly aimed at the side view mirror of the vehicle. Additionally, 2 G.R.A.S. reference microphones was set inside the car, one was near the side view mirror triangle as microphone 1, the other was at the driver's ear position as microphone 2 (Fig. 2). The signals of the array and two reference microphones were recorded simultaneously. 


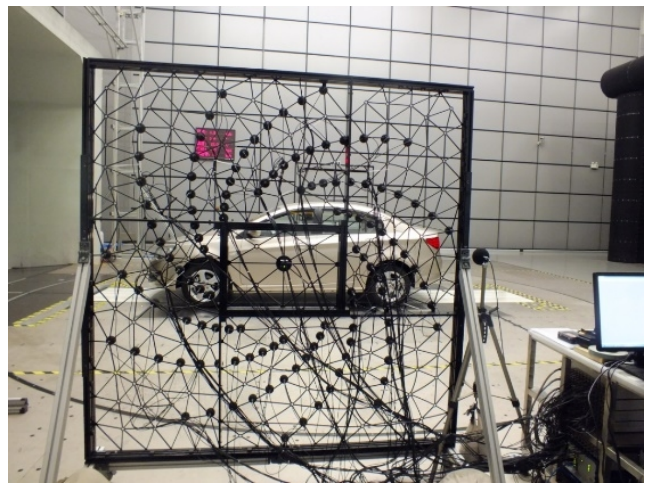

Fig. 1 120-channel planar spiral array positioned out-of-flow of AAWT

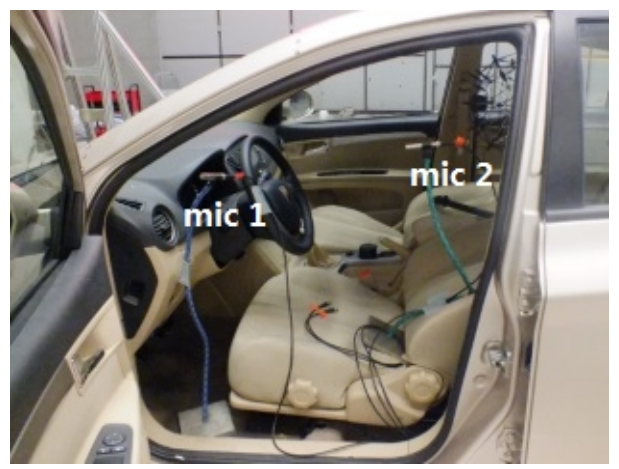

Fig. 22 reference microphones inside the vehicle

\section{Results and Analysis}

Exterior Sound Sources Identification. With the help of HDR algorithm, the exterior sound sources under different yaw angles were identified. As shown in Fig.3, no matter under which condition, the exterior main source always locates in the front wheel region. As the yaw angle increases from $-20^{\circ}$ to $+20^{\circ}$, the sound pressure level of the main source decreases from $88.92 \mathrm{dBA}$ to 86.31dBA. Except for the wheel region, the side view mirror is most interested. Just like the front wheel region, as the yaw angle increases, the noise at the side view mirror becomes weaker. Even under the yaw angle of $+20^{\circ}$, the side view mirror can't be identified. The reason is that, as yaw angle with minus degree of $-20^{\circ}$, airflow separation and pressure fluctuation around vehicle body surface near the driver ear position becomes stronger, so makes greater noise level.

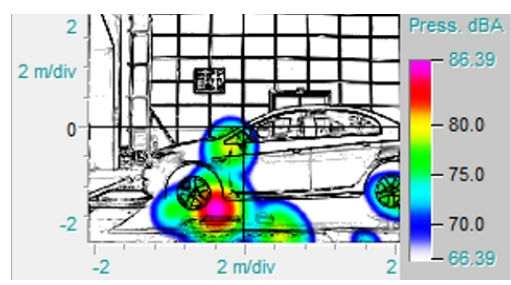

a) Yaw angle: $0^{\circ}$

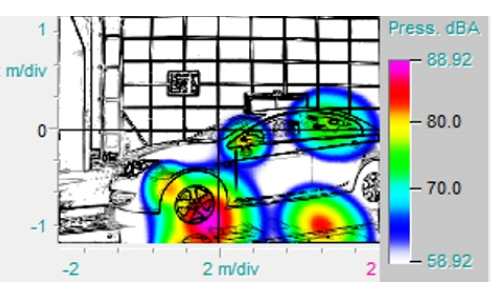

b) Yaw angle: $-20^{\circ}$

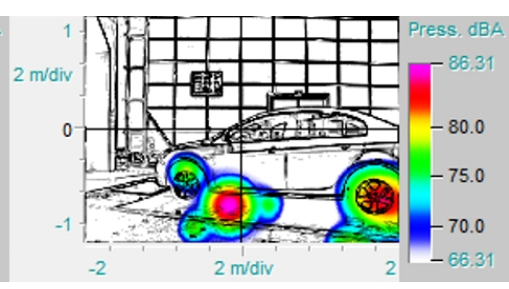

c) Yaw angle: $+20^{\circ}$

Fig.3 Vehicle exterior acoustic map under different yaw angles

Correlation analysis. With Eq. 2 cross correlation function in the time domain was calculated between exterior array signals and interior microphone 1. Although side view mirror is not the main exterior source, it plays a dominant role for the interior noise, as shown in Fig.4. Not only for microphone 1, for driver's ear position, the same conclusion can be drawn. The maximum correlation can be $0.75 \%$ under the yaw angle of $0^{\circ}$. The correlation under the yaw angle of $-20^{\circ}$ is only $0.30 \%$, which is lower than the $0.35 \%$ under the yaw angle of $+20^{\circ}$. This phenomenon is far from expectation, 
because the side view mirror can even not be identified as an exterior source under the yaw angle of $+20^{\circ}$. The reason can be speculated that under the yaw angle of $-20^{\circ}$, not only the side mirror, the noise of front wheel region and other sources also increased remarkably due to the much stronger turbulence. So the contribution of the side view mirror relatively decreases. That means it's not reasonable to use an absolute number of correlation to evaluate the influence of exterior sound source to inner noise. Only the correlation comparison of different exterior sources in the same condition makes sense.

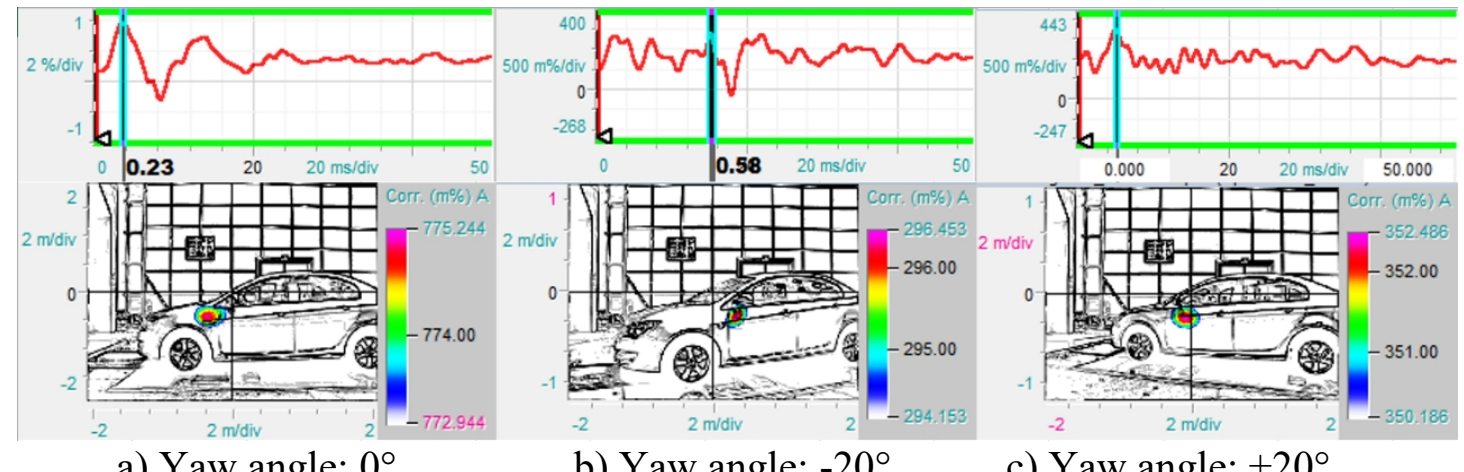

a) Yaw angle: $0^{\circ}$

b) Yaw angle: $-20^{\circ}$

c) Yaw angle: $+20^{\circ}$

Fig.4 Correlation of exterior sound source and microphone 1 under different yaw angles

No matter with which microphone, the origin source is always the side view mirror. Usually the correlation with microphone 2 is lower than microphone 1 due to the further transmission path.

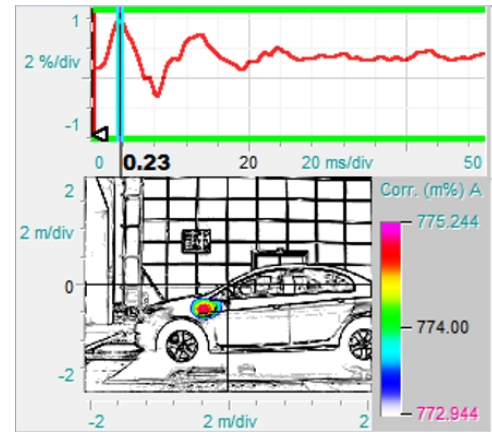

a) Microphone 1

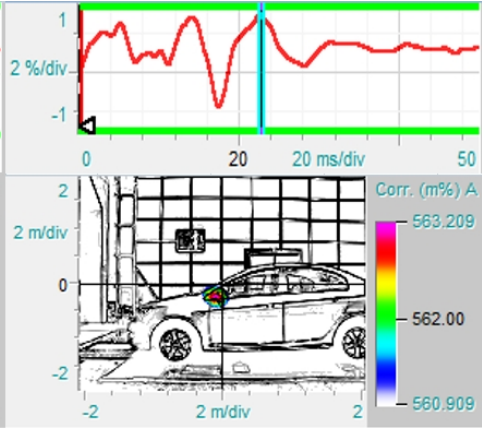

b) Microphone 2

Fig. 5 Correlation with 2 microphones under the yaw angle of $0^{\circ}$

\section{Conclusions}

In this study, exterior sound sources were identified under different yaw angles. According to the correlation analysis, the sound source which makes the most contribution to reference microphone inside the vehicle is distinguished out. The main exterior source (front wheel region) is not dominant for interior noise level, while instead of it the side view mirror plays the most important role, which can guide designers and engineers to pay more attention to the shape of side view mirror and the transmission path optimization.

\section{Acknowledgements}

This work was financially supported by State Key laboratory of Vehicle NVH and Safety Technology (NVHSKL-201403). 


\section{References}

[1] George A R. Automobile aerodynamic noise[R]. SAE Technical Paper, 1990.

[2] Dougherty R P. Noise source imaging by beamforming[R]. SAE Technical Paper, 2008.

[3] Christensen J J, Hald J. Technical review beamforming[J]. Bruel\&Kjear, Danmark, 2004: 3-12.

[4] Neugebauer, Stefan, Reinhard Rösel, and Dirk Döbler: submitted to Internoise (2014).

[5] Yinzhi, He, Zhigang Yang, and Yigang Wang: submitted to Proceedings of the FISITA 2012 World Automotive Congress (2013) . 